Original Research Article

\title{
Feeding practices and problems in breastfeeding in the first postnatal week in late preterm infants
}

\author{
Harsha P. J. ${ }^{1}$, Roop Kumar C. ${ }^{2}$ \\ ${ }^{1}$ Dr Harsha P J, Assistant Professor, ${ }^{2}$ Dr Roop Kumar C, Senior Resident, both authors are affiliated with \\ Department of Pediatrics, PES Institute of Medical Sciences and Research, Kuppam, Andhra Pradesh, India.
}

Address for Correspondence: Dr Harsha P.J., Assistant Professor, Department of Pediatrics, PES Institute of Medical Sciences and Research, Kuppam, Andhra Pradesh, India. Email:harshabattery@gmail.com

\begin{abstract}
Background and objective: Breastfeeding problems exist despite advice and counseling. Problems are more in late preterm babies compared to term. Problems in feeding start in early postnatal life which continues later. The aim of the study was done to know the feeding practices and problems in breastfeeding in the first postnatal week in late preterm babies. Methods: This was a cross sectional study. Total 77 late preterm babies were included in the study. Results: Only $28.57 \%$ initiated breast feeding within one hour of birth. Initiation of breast feeding within one hour after birth and exclusive breastfeeding in first postnatal week was seen better in late preterm babies born by vaginal route, born to mulitparous and male sex. Prelacteal feeds were given in $16.88 \%$. Bottle feeding was seen in $62.75 \%$ in mothers who did not follow exclusive breast feeding in the first postnatal week. Most common causes for non-exclusive breast feeding in first postnatal week was insufficient milk (72.55\%), baby not able to suck (60.78\%), elders advice (54.9\%) and wound pain (27.45\%). Problems with breast feeding are more common in late preterm infants born by Cesarean Section. Conclusion: Despite counseling in antenatal and postnatal period, there was delayed initiation of breast feeding. Problems of mixed feeding, predominant non breast feeding, bottle feeding and practice of giving prelacteal feeds still continues.
\end{abstract}

Keywords: Breastfeeding, Initiation, Late Preterm Infant, Non-exclusive breastfeeding.

\section{Introduction}

Timely initiation of breastfeeding can help to prevent neonatal mortality caused by infections such as sepsis,pneumonia anddiarrhea [1,2].Breastfeeding provides an ideal food for healthy growth and development of infants and children. There is a current trend of early discharge of mother and newbornfrom the hospital after delivery, often breastfeeding issues are not addressed properly and may lead to low Exclusive breastfeeding (EBF) rates.

There is a paucity of data from developing countries on this issue. Several studies focusing on the constraints to EBF concluded that; breastfeeding problems, delivery by caesarean section, perceived or real breast milk insufficiency, inadequate weight gain of the infant, resumption of official work by the

Manuscript received: $5^{\text {th }}$ September 2017 Reviewed: $15^{\text {th }}$ September 2017

Author Corrected: $24^{\text {th }}$ September 2017

Accepted for Publication: $30^{\text {th }}$ September 2017 mother, and cultural practices are the factors influencing mothers' decision for continuation of breastfeeding [3]. The bioactive components of breast milk can prove especially crucial for LPIs (Late Preterm Infants) who "have a compromised immunomodulatory response, have immature organs including the brain, and are susceptible to inflammatory injury and oxidative stress"[4]. LPIs have lower rates of exclusive breastfeedingthan both term infants and early term infants (37-38 weeks gestational age), in part, as families do not receive sufficient and appropriate support in the immediate postpartum period [5]. Compared with infants initiating breastfeeding within the first hour of life, neonatal mortality between enrolment and 28 days was higher in infants initiating at $2-23 \mathrm{~h}$ and in those initiating at 24-96 h. These associations were similar when deaths in the first 4 days of life were excluded [6]. Hence this study was done to know the feeding practices in first postnatal week in LPIs. 


\section{Material and Methods}

Objective- To know the feeding practices and problems in breastfeeding in the first postnatal week in late preterm infants

Inclusion Criteria- Late preterm infants $\left(\geq 34^{0 / 7}\right.$ $36^{6 / 7}$ weeks of gestational age) who did not require admission to NICU (Neonatal intensive care unit) in the first postnatal week

Exclusion criteria- Neonates with any chromosomal / congenital anomalies (e.g cleft lip/ palate, retrognathia etc.), any contraindication to breastfeeding (HIV infection and treatment with anticancer drugs), multiple gestations, acutely ill mothers, babies requiring admission to NICU and mothers who did not give consent for the study.

Study Design- Across sectional study conducted from June 2015 to November 2015.

Setting- Department of Paediatrics, PES Institute of Medical Sciences and Research, Kuppam, Andhra Pradesh

Participants and source of data- The mothers of late preterm babies were interviewed on follow up for vaccination at 6 weeks of age at OPD (Out Patient Department).

Methods- A face to face interview was conducted with mothers of late preterm infants on follow up at 6 weeks for immunization in OPD. Answer to questions on feeding was noted on recall basis. The mothers were asked about initiation of breast feeding after birth and classified into $<1$ hour, 2-23 hours and 24-96 hours. The details such as mode of delivery (vaginal/ cesarean section), multi/ primiparous and sex of baby (male/ female) was noted. History of prelacteal feeds and if given, nature of prelacteal feeds given was noted. The type of feeding in the first postnatal week such as only breast milk, mixed feeding or predominant cow's/formula milk/both was noted. The problems for not giving exclusive breastfeeding in first postnatal week were noted. The initiation of breastfeeding and feeding practices in first postnatal week was analyzed for sex of baby, mode of delivery (vaginal/ cesarean section) and parity of mother (multi/primiparous).

Statistical Methods- The results were entered in microsoft excel sheet and expressed as percentage.

\section{Results}

Total 77 mothers of late preterm babies who did not require admission to NICU were included in the study. All babies were delivered in hospital. There was only one pair of late preterm twin and excluded from the study. Counseling regarding breastfeeding was received in $79.22 \%(61 / 77)$ during antenatal check up. Total 58(75.32\%) were born by vaginal route and $19(24.68 \%)$ through cesarean section. Total $53(68.83 \%)$ were born to multiparous mother and 24(31.17\%) to primiparous. Male babies were $42(54.55 \%)$ and female $35(45.45 \%)$.

Feeding practices seen in the first postnatal week in late preterm infants are shown in Table 1.

Initiation of breastfeeding within one hour after birth was seen better in late preterm babies born through vaginal delivery $(32.76 \%, 19 / 58)$ compared to born by cesarean section $(15.79 \%, 3 / 19)$, with multiparous mother's $(33.46 \%, 18 / 53)$ compared toprimiparous's mother $(16.67 \%, 4 / 24)$ and male babies $(30.95 \%, 13 / 42)$ compared to female babies $(25.71 \%, 9 / 35)$.

Practice of giving prelacteal feeds was seen in $16.8 \%$ (Table 1). In all cases was initiated by elders in the family as a traditional belief and nature of prelacteal feeds given is shown in Table-1.

Exclusive breastfeeding in the first postnatal week was seen in only $33.77 \%$ (Table 1 ).

Bottle feeding was practiced in 62.75\% (32/51) who did not follow (n-51) exclusive breastfeeding in first postnatal week. Pallada or spoon feeding was seen in $37.25 \%(19 / 51)$. Only $16.88 \%(13 / 77)$ consulted doctor or health care personnel about feeding problems and advice after birth on their own apart from routine counseling from health care personnel which all babies received. False feeding practices were followed despite feeding advice by health care personnel. 
Table-1: Feeding practices in the first postnatal week in late preterm infants.

\begin{tabular}{|l|c|}
\hline Initiation of breastfeeding after birth & $28.57 \%(22 / 77)$ \\
$<1$ hour & $62.34 \%(48 / 77)$ \\
$2-23$ hours & $9.09 \%(7 / 77)$ \\
$24-96$ hours & $16.88 \%(13 / 77)$ \\
\hline Prelacteal feeds & $15.38 \%(2 / 13)$ \\
Plain water & $23.08 \%(3 / 13)$ \\
Sugar water & $38.46 \%(5 / 13)$ \\
Cow's Milk & $15.38 \%(2 / 13)$ \\
Formula Milk & $7.69 \%(1 / 13)$ \\
Others (both cow's milk and formula milk) & $33.77 \%(26 / 77)$ \\
\hline Type of breastfeeding in first postnatal week & $57.14 \%(44 / 77)$ \\
Only breast milk & $9.09 \%(7 / 77)$ \\
Mixed feeding & \\
Predominant non-breastfeeding (formula feeds /cow's milk/both) & $72.55 \%(37 / 51)$ \\
\hline Reasons for non-exclusive breastfeeding in first postnatal week & $60.78 \%(31 / 51)$ \\
Insufficient & $54.09 \%(28 / 51)$ \\
Not able to suck & $27.45 \%(14 / 51)$ \\
Elders advice & $13.73 \%(7 / 51)$ \\
\hline Wound Pain & \\
Breast and nipple problems & \\
\hline
\end{tabular}

Most common causes for non-exclusive breastfeeding in the first postnatal week in late preterm babies was insufficient milk (72.55\%), baby not able to suck (60.78\%), elders advice (54.9\%) andwound pain (27.45\%). Out of 14 with wound pain as hindrance to breastfeeding in first postnatal week, $11(78.57 \%)$ of mothers had delivered by cesarean section. In late preterm babies mothers not giving exclusive breastfeeding in the first postnatal week was seen more common in those born by cesarean section $(78.95 \%, 15 / 19)$ compared tothose born byvaginal route $(62.07 \%, 36 / 58)$, primiparous mother's $87.5 \%(21 / 24)$ compared to mulitparous $56.60 \%(30 / 53)$ and female babies $(68.57 \%, 24 / 35)$ compared to female babies $(64.29 \%, 27 / 42)$.

\section{Discussion}

Total 77 late preterm babies were included in the study. Babies born to primiparous mothers were $68.83 \%$. Late Preterm babies delivered by vaginal route were $75.32 \%$. Male babies were $54.55 \%$. S Suresh et al included babies born at or after 34 weeks of gestational age in his study $(n=400)$. Total $54 \%$ were male, born to primiparous were $66 \%$, delivered vaginally were $63.3 \%$ [3].

In our study initiation of breastfeeding $<1$ hour was observed in $28.57 \%, 2-23$ hour in $62.34 \%, 24-96$ hours in $9.09 \%$. The initiation of breastfeeding is vital to achieving the WHO's recommendation $[7,8]$ as it has been found to be associated with longer-term breastfeeding and lower infant mortality, particularly in developing countries $[9,10]$. Initiation of breast feeding within one hour after birth was $60 \%$ and $50.3 \%$ in different studies $[11,12]$. Early or timely initiation of breastfeeding, specifically within $1 \mathrm{~h}$ of birth, refers to the best practice recommendation by the World Health Organization (WHO) [13]. A recent systematic review and meta-analysis revealed that breastfeeding initiation after the first hour of birth doubles the risk of neonatal mortality [14]. Several South Asian countries have the worst early initiation of breastfeeding practices in the world.

The rates in Pakistan, India, Bangladesh and Nepal are only $29 \%, 41 \%, 47 \%$ and $45 \%$ respectively [15]. Prelacteal feeds was given in $16.88 \%$. Cows milk (38.46\%), sugar water $(23.08 \%)$ was most commonprelacteal feeds given. Practice of giving prelacteal feeds exist despite counseling on breast 
feeding during antenatal and postnatal period as traditional belief that colostrums is not good for health. According to one studyin Amhara region, that colostrum discarding and pre-lacteal feeding practices were challenges to timely initiationof breastfeeding [16].

Exclusive breast feeding in first postnatal week was given in only $33.77 \%$ in this study. Mixed feeding was seen in $57.14 \%$ and predominant non-breast feeding in $9.09 \%$.

Most common cause for non exclusive breast feeding in first postnatal week in late preterm babies were feeling of insufficient milk (72.55\%), not able to suck at breast $(60.78 \%)$, elders advice $(54.09 \%)$, Wound pain (27.45\%)andbreast and nipple problems $(13.73 \%)$ in decreasing order of frequency.

The problems of initiation of breast feeding within one hour after birth and non exclusive breast feeding was seen in babies born by cesarean section, primiparous mothers and female babies. Problem of woundpain as hindrance to breastfeeding in first postnatal week was seen in $78.57 \%$ of mothers who had delivered by cesarean section.

Breastfeeding problems were present in $88.5 \%$ of newborn before discharge. The major problems were poor positioning and attachment (88.5\%), followed by breast and nipple problems (30.3\%). Breastfeeding issues were noted in $72.5 \%$ during follow-up.

Most commonly identified breastfeeding problems were poor positioning and attachment (70.3\%), sore or cracked nipple (17.8\%) followed by retracted or flat nipple (15\%) and breast engorgement (13\%).

Major factors associated with occurrence of breastfeeding problems were multiparity, lack of previous experience of breastfeeding and cesarean delivery. The only independent risk factor for breastfeeding problems in multivariate analysis was caesarean delivery. EBF rate at 6 months was $69.5 \%$.

Major reasons given by the mothers for discontinuation of breastfeeding were concern ofpoor weight gain in baby (28.6\%), advice from elders athome $(27.7 \%)$ and perception of breast milk insufficiency $(25 \%)$. Few mothers reported reasons like breastfeedingproblems $(8 \%)$, baby refused to suck or had suckingdifficulty $(7.1 \%)$ and baby remains hungry after feeding $(3.6 \%)$ as a reason for non-EBF[3].

There is a high prevalence of breastfeeding problemsin newborndelivering after 34 weeks of gestation. An interesting observation was the persistence of these problems at the end of 1 st week (poor positioning and attachment $-70.8 \%$ ) and increase in some reported problems (Sore or cracked nipple from $10.5 \%$ to $17.8 \%$ and breast engorgement $4.8-13 \%$ ) after discharge.

There is a requirement of focused lactation counseling and support for these mothers [3]. Mothers delivered by cesarean section weremore likely to report breastfeeding problems comparedwith those delivered vaginally. Other studies also haveshown similar results $[3,17]$.

Late preterm infantswho have difficulty latching on tothe breast correctly could likely have immature suckingand swallowing reflexes. ${ }^{(18)}$ Parents and health care personnel and doctorsshouldunderstand the unique feeding challenges due to the relationshipbetween gestational age and brain development.

\section{Conclusion}

The problems of breastfeeding such as notinitiating breastfeeding within one hour after birth, non exclusive breastfeeding in first postnatal week was seen more in babies born by cesarean section, born to primiparous and female sex. Problems such as giving prelacteal feeds, bottle feeding exists despite breastfeeding counseling.

There should be more vigorous counseling aboutlactation problems, uses of breast milk, harmful practices and support in early postnatal period for breast feeding.

Limitation of the study- This study was done only on feeding practices and problems of breastfeeding in late preterm infants and was not compared with term infants. Follow up was not done in this study.

What does the study add to existing knowledge? Problems in breast feeding and harmful feeding practices exist in late preterm babies despite antenatal counseling.There is need for strengthening 
of antenatal and postnatal counseling on breastfeeding, harmful feeding practices. Health care organizations should look at the gap between actual knowledge on breastfeeding and the practice followed in that area to orient the health service providers.

Funding: Nil, Conflict of interest: None initiated, Perission from IRB: Yes

\section{References}

1. Lawn JE, Kerber K, Enweronu-Laryea C, Massee Bateman O. Newborn survival in low resource settings--are we delivering? BJOG. 2009 Oct;116 Suppl 1:49-59. doi: 10.1111/j. 1471-0528. 2009. 02328.x.

2. Alive and Thrive. Insight: Impact of early initiation of exclusive breastfeeding on newborn death. Washington DC: A \& $\mathrm{T}$ Technical Brief. Issue 1. 2010.

3. Sithara Suresh, Kamlesh K. Sharma, Manju Saksena, AnuThukral, Ramesh Agarwal, Manju Vatsa. Predictors of Breastfeeding Problems in The First Postnatal Week and Its Effect on Exclusive Breastfeeding Rate at Six Months: Experience in A Tertiary Care Centre in Northern India. Indian Journal of Public Health. 2014; 58(4): $270-73$.

4. Meier P, Patel AL, Wright K, Engstrom JL. Management of breastfeeding during and after the maternity hospitalization for late preterm infants. ClinPerinatol. 2013 December;40(4):689-705. doi:10.1016/j.clp.2013.07.014.

5. Goyal NK, Attanasio LB, Kozhimannil KB. Hospital care and early breastfeeding outcomes among late preterm, early-term, and term infants. Birth. 2014;41(4):330-8.doi: 10.1111/birt.12135. Epub 2014 Oct 8.

6. NEOVITA Study Group. Timing of initiation, patterns of breastfeeding, and infant survival: prospective analysis of pooled data from three randomised trials. Lancet Glob Health 2016 Apr;4 (4):e266-75. doi: 10. 1016 / S2214-109X (16) 00040-1.

7. DiGirolamo AM, Grummer-Strawn LM, Fein SB. Effect of maternity-care practices on breastfeeding.
Pediatrics. 2008;122(2):S43-9.doi: 10.1542/peds. 2008-1315e.

8. Moore ER, Anderson GC, Bergman N, Dowswell T. Early skin-to-skin contact for mothers and their healthy newborn infants. Cochrane Database Syst Rev. 2012 May 16;(5):CD003519. doi: 10.1002/ 14651858.CD003519.pub3.

9. Lawn JE, Cousens S, Zupan J. 4 million neonatal deaths: when? Where? Why? Lancet. 2005;365 (9462): 891-900. DOI: http://dx.doi.org /10.1016 /S0140-6736(05)71048-5.

10. Getachew Tilahun, Getu Degu, TelakeAzale and AskalTigabu. Prevalence and associated factors of timely initiation of breastfeeding among mothers at DebreBerhan town, Ethiopia: a crosssectional study. International Breastfeeding Journal.2016;11:27. DOI 10.1186/s13006-016-0086-5

11. Tegegn Tadesse, FirehiwotMesfin and Tefera Chane. Prevalence and associated factors of nonexclusive breastfeeding of infants during the first six months in rural area of Sorro District, Southern Ethiopia: a cross-sectional study. International Breastfeeding Journal.2016;11:25. DOI 10.1186/s 13006-016-0085-6

12. WHO. Breastfeeding-early initiation: World Health Organization; 2012 [updated 2012]. http: //www.who.int/elena/titles/early_breastfeeding/en/A ccessed 5 April 2013.

13. Khan J, Vesel L, Bahl R, MartinesJC. Timing of breastfeeding initiation and exclusivity of breastfeeding during the first month of life: effect sonneonatal mortality and morbidity--asystematic reviewandmeta-analysis. Matern Child Health J.2015 Mar;19(3):468-79. doi: 10.1007/s 10995014-1526-8.

14. UNICEF. State of the World's Children 2014: every child counts. New York:United Nations Children's Fund (UNICEF); 2014.

15. Rogers NL, Abdi J, Moore D, Nd'ianguiS, Smith LJ, Carlson AJ, CarlsonD. Colostrumavoidance, prelacteal feeding and late breast-feeding initiation inrural Northern Ethiopia. Public Health Nutr.2011 Nov;14(11): 2029-36. doi: 10.1017/ S13689800 11000073. Epub2011 Apr 21. 
Original Research Article

16. Schluter PJ, Carter S, Percival T. Exclusive and any breastfeeding rates of Pacific infants in Auckland: Data from the Pacific Islands Families First Two Years of Life Study. Public Health Nutr 2006; 9 (6):692-9.
17. Santos IS, Matijasevich A, Silveira MF, Sclowitz IK, Barros AJ, Victora CG, Barros FC. Associate dfactors and consequences of latepreterm births: results from the 2004 Pelotasbirthcohort. Paediatr Perinat Epidemiol. 2008 Jul; 22(4):350-9. doi: 10.1111/j.1365-3016.2008.00934.x.

How to cite this article?

Harsha P. J, Roop Kumar C. Feeding practices and problems in breastfeeding in the first postnatal week in late preterm infants. J PediatrRes. 2017;4(09):546-551.doi:10. 17511/ijpr.2017.i09.02. 